\title{
Prevalence of 7 virulence genes of Legionella strains isolated from environmental water sources of public facilities and sequence types diversity of $L$. pneumopila strains in Macau
}

\author{
Lina Xiong ${ }^{1,2}$, Hongbo Zhao ${ }^{2}$, Ziyao $\mathrm{Mo}^{2, *}$, Lei Shi ${ }^{1}$ \\ ${ }^{1}$ School of Light Industry and Food Sciences, South China University of Technology, Guangzhou, Guangdong, China; \\ ${ }^{2}$ The First Affiliated Hospital of Guangzhou Medical University, Guangzhou Institute of Respiratory Disease, State Key \\ Laboratory of Respiratory Disease, Guangzhou, Guangdong, China.
}

\begin{abstract}
Summary
In this study, we analyzed 7 virulence genes in 55 Legionella species (including 29 L. pneumophila and 26 non- $L$. pneumophila strains) which isolated from environmental water sources of the public facilities in Macau by using PCR and real-time PCR. In addition, 29 Legionella pneumophila isolates were subjected to genotyping by sequence-based typing scheme and compared with the data reported. The detection rate of flaA, pilE, asd, mip, mompS, proA and neuA genes in the $L$. pneumophila were $100.0 \%$, respectively. The neuA gene was not detected in the non- $L$. pneumophila strains, but flaA, pilE, asd, mip, mompS, and $\operatorname{pro} A$ genes could be amplified with a positive rate of $15.4 \%, 15.4 \%, 53.8 \%, 38.5 \%$, $15.4 \%$, and $38.5 \%$, respectively. The results from real-time PCR were generally consistent with that of PCR. Those $L$. pneumophila strains were assigned into 10 sequence types (STs) and ST1 (9/29) was the dominant STs. Four new STs were found to be unique in Macau. The analysis of population structure of $L$. pneumophila strains which isolated from Macau, Guangzhou and Shenzhen indicated that the similar clones were existed and ST1 was the most prevalent STs. However, the distribution of the subtypes isolated from Macau was not the same extensive as those from Guangzhou and Shenzhen. The different detection rates of the 7 virulence genes in different species of Legionella might reflect their own potential for environmental adaptability and pathogenesis. And the data analyzed from STs diversity indicated the Macau $L$. pneumophila possessed obvious regional specificity and high genetic diversity.
\end{abstract}

Keywords: Sequence-based typing, population structure, phylogenetic relationship

\section{Introduction}

Legionella species, commonly found in the environment, are the major causative agents of Legionnaire's disease and Pontiac fever. They have been found to not only induce lung infection, but also to cause dysfunction of other organs, such as the heart, kidney and central nervous system (1). To date, more than 50 species of

\footnotetext{
*Address correspondence to:

Dr. Ziyao Mo, The First Affiliated Hospital of Guangzhou Medical University, Guangzhou Institute of Respiratory Disease, State Key Laboratory of Respiratory Disease, No. 151, YanJiangXi Road, YueXiu District, Guangzhou 510120, Guangdong, China.

E-mail:moziyao@gird.cn
}

Legionella have been described (2). Among of them, 20 species are recognized as human pathogens. $L$. pneumophila was identified as primary culprit for Legionnaire's disease. In recent years, several studies related to the presence of Legionella have been reported in southern Chinese cities, such as Guangzhou, Shenzhen, Jiangmen and Hong Kong $(3,4)$. However, no data of Legionella from Macau were published. In May of 2010, a case of Legionella infection emerged, which was vigorously suspected to be caused by local Legionella species since the patient did not previously travel abroad. We investigated and detected the existence of Legionella in natural and artificial water environments in Macau in the summer (from May to July) of the same year. A total of 55 isolates of Legionella were isolated from air conditioning cooling towers, fountains and 
surface waters in public facilities of Macau (5).

In the present study, seven virulence genes ( $f a A$, pile, asd, mip, mompS, proA and neuA) responsible for the expression of adherence, invasion, colonization and cytotoxin production $(6,7)$, were detected in all 55 strains isolated from Macau. For comparison, the PCR and the real-time PCR were applied to detect the genes simultaneously. In addition, sequence-based typing (SBT), a powerful epidemiological method recognized by the European Working Group for Legionella Infections (EWGLI) as a "gold standard" tool, was used for the L. pneumophila strains. The population structure and phylogenetic relationship of L. pneumophila strains isolated from Macau were analyzed and compared to those from near cities such as Guangzhou and Shenzhen. This investigation will help us to learn more about the characteristics of the Macau Legionella isolates, provide the possibility to trace and control for a large area of epidemic and appropriate precaution strategy against Legionella infection.

\section{Materials and Methods}

\subsection{Legionella strains}

A total of 55 Legionella strains were collected from 43 water samples including air conditioning cooling towers (27 samples), fountains (13 samples) and surface waters (3 samples) in public sites of Macau. Legionella species were identified by serological agglutination with Legionella Latex Agglutination Kit (PRO-LAB, Weston, USA) based on manufacturer instructions and fatty acid analysis was performed with the Sherlock microbial identification system (software version 6.0, MIDI, USA; Microbial ID, Inc., Newark, Del).

\subsection{Preparation of DNA}

The strains were cultured with buffered charcoal yeast extract (BCYE) ager plates at $37^{\circ} \mathrm{C}$ in $5 \% \mathrm{CO}_{2}$. A single colony of Legionella was picked from the plate and resuspended into $100 \mu \mathrm{L}$ sterilized ultrapure water. DNA was then extracted by one thaw-freeze cycle $\left(99^{\circ} \mathrm{C}\right.$ for $10 \mathrm{~min}$ and $4^{\circ} \mathrm{C}$ for $\left.5 \mathrm{~min}\right)$. After briefly centrifuged, the supernatant was measured with a spectrophotometry at $260 \mathrm{~nm}$ in triplicates using A260/ A280 ratio (NanoDropTM 1000, Thermo Scientific) then used as the DNA template for PCR and real-time PCR.

\subsection{Detection of pathogenic genes by PCR and real- time PCR}

The PCR primers were based on EWGLI recommended $(8,9)$. PCR conditions were the same as described by Gaia et al. (8) with minor modification. PCR was performed in a mixture $(50 \mu \mathrm{L})$ consisting of $25 \mu \mathrm{L}$ of
$2 \times$ SuperStar PCR mix (GenStar Biosolution, Beijing, China), 5 pmol each primers, $100 \mathrm{ng}$ of the template. Sterile distilled water was added to make $50 \mu \mathrm{L}$. The products were detected by electrophoresis and purified using the DNA Gel Extraction Kit (Axygen USA).

To establish a more sensitive, higher speciality and easier operation detecting method, a real-time PCR was applied at the present study. The primers used in real-time PCR were designed based on sequences from GenBank accession numbers X83232 (flaA), AF048690 (pilE), AF034213 (asd), AJ496269 (mip), AF078136 (mompS), M32884 (proA) and AJ007311 (nеuA). The primers sequences were list in Table S1. For amplifications, $50 \mathrm{ng}$ template was mixed with 12.5 $\mu \mathrm{L} 2 \times$ SYBR Premix EX Taq ${ }^{\mathrm{TM}}$ II (Perfect Real Time, Takara, Japan), and 5 pmol each of the forward and the reverse primers in a final volume of $25 \mu \mathrm{L}$. The reaction conditions were $5 \mathrm{~s}$ at $95^{\circ} \mathrm{C}$ and $30 \mathrm{~s}$ at $60^{\circ} \mathrm{C}$ for 40 cycles. Sterilized water was used as a template for the blank control for both PCR reactions.

\subsection{Sequence-based typing}

The purified PCR products were sequenced by Beijing Genomics Institute (Beijing, China). SBT using loci flaA, pile, asd, mip, mompS, proA and neuA was performed according to the EWGLI scheme $(8,9)$. Genotype analysis was based on the standard SBT method given by the EWGLI with these 7 genes. The nucleotide sequences obtained were confirmed by the SBT database available on the EWGLI website (http:// www.ewgli.org/), and the sequences were compared with those in the SBT database from the website (http:// www.hpabioinformatics.org.uk/legionella/legionella sbt/php/sbt_homepage.php).

\subsection{Population structure and phylogenetic analysis}

The minimum spanning trees (MST) were conducted by the BioNumerics software (version 7.1; Applied Maths, Kortrijk, Belgium). In MST, the ST that possesses the most number of single-locus variants is defined as the founder ST. The clusters of relative STs that originate from a common ancestor are considered as the clone groups or complexes. The single genotype that does not correspond to any other clone groups is classified as singleton. The sequence types are represented by the circles. The size of circle indicates the number of the particular strains. The relationship of the different circles is present with the connecting lines.

The concatenated sequence in phylogenetic analysis was prepared with the seven loci of the initial SBT scheme according to their locations on the chromosome by BioEdit (http://www.mbio.ncsu.edu/BioEdit/ bioedit.html). Based on the concatenated sequence, the evolutionary relationship between STs was conducted using the neighbour-joining method with Tamura 
Table 1. Detection rate of seven genes in Legionella strains isolated from Macao

\begin{tabular}{|c|c|c|c|c|c|c|c|c|c|c|c|c|c|c|c|}
\hline \multirow{3}{*}{ Species } & \multirow{3}{*}{$\begin{array}{l}\text { No. of } \\
\text { strains }\end{array}$} & \multicolumn{12}{|c|}{ No. of positive strains } & & \\
\hline & & \multicolumn{2}{|c|}{ flaA } & \multicolumn{2}{|c|}{ pile } & \multicolumn{2}{|c|}{ asd } & \multicolumn{2}{|c|}{ mip } & \multicolumn{2}{|c|}{ mompS } & \multicolumn{2}{|c|}{ proA } & \multicolumn{2}{|c|}{ пеиA } \\
\hline & & $\mathrm{P}^{\mathrm{a}}$ & $\mathrm{R}^{\mathrm{b}}$ & $\mathrm{P}$ & $\mathrm{R}$ & $\mathrm{P}$ & $\mathrm{R}$ & $\mathrm{P}$ & $\mathrm{R}$ & $\mathrm{P}$ & $\mathrm{R}$ & $\mathrm{P}$ & $\mathrm{R}$ & $\mathrm{P}$ & $\mathrm{R}$ \\
\hline L. pneumophila & 29 & 29 & 27 & 29 & 29 & 29 & 29 & 29 & 28 & 29 & 29 & 29 & 29 & 29 & 29 \\
\hline L. adelaidensis & 1 & 0 & 0 & 0 & 0 & 0 & 0 & 0 & 0 & 0 & 0 & 0 & 0 & 0 & 0 \\
\hline L. rubrilucens & 3 & 1 & 0 & 0 & 0 & & 0 & 0 & 0 & 1 & 1 & 0 & 0 & 0 & 0 \\
\hline fluoribacter-gormanii & 3 & 0 & 0 & 2 & 1 & 3 & 0 & 0 & 0 & 0 & 0 & 0 & 0 & 0 & 0 \\
\hline L. shakespearei & 5 & 0 & 0 & 0 & 2 & 5 & 4 & 5 & 3 & 1 & 3 & 5 & 4 & 0 & 3 \\
\hline L. feeleii & 6 & 0 & 0 & 0 & 0 & 0 & 0 & 0 & 0 & 1 & 4 & 0 & 0 & 0 & 2 \\
\hline L. wadsworthii & 3 & 2 & 2 & 0 & 0 & 3 & 1 & 0 & 0 & 0 & 0 & 1 & 3 & 0 & 0 \\
\hline L.quateirensis & 5 & 1 & 0 & 2 & 2 & 3 & 0 & 5 & 3 & 1 & 4 & 5 & 3 & 0 & 0 \\
\hline \multicolumn{16}{|c|}{ Detection rate of seven genes with three different methods $(\%)$} \\
\hline L. pneumophila & 29 & 100 & 93.1 & 100 & 100 & 100 & 100 & 100 & 96.5 & 100 & 100 & 100 & 100 & 100 & 100 \\
\hline No-L. pneumophila & 26 & 15.4 & 7.69 & 15.4 & 19.2 & 53.8 & 19.2 & 38.5 & 23.1 & 15.4 & 46.2 & 42.3 & 38.5 & 0 & 19.2 \\
\hline
\end{tabular}

${ }^{\mathrm{a} P C R} ;{ }^{\mathrm{b}}$ Real-time PCR.

Table 2. Sequences-Based Typing of $L$. pneumophila strains from Macao

\begin{tabular}{|c|c|c|c|c|c|c|c|c|c|}
\hline SBT type & flaA & pile & asd & mip & mompS & proA & пеи & Serotype (no.) & No. of isolate \\
\hline 1 & 1 & 4 & 3 & 1 & 1 & 1 & 1 & Lp1(5)LP14(4) & 9 \\
\hline 160 & 11 & 14 & 16 & 16 & 15 & 13 & 9 & Lp1(1)Lp14(1) & 2 \\
\hline 566 & 16 & 4 & 3 & 1 & 1 & 1 & 1 & Lp1(2)Lp14(1) & 3 \\
\hline 752 & 22 & 4 & 3 & 1 & 1 & 1 & 1 & Lp1 & 1 \\
\hline 1119 & 2 & 10 & 14 & 10 & 21 & 4 & 3 & Lp1 & 2 \\
\hline 1417 & 8 & 6 & 34 & 9 & 2 & 8 & 209 & Lp14 & 4 \\
\hline $01^{\mathrm{a}}$ & 11 & 14 & 16 & 25 & 15 & 13 & 206 & Lp14 & 1 \\
\hline $02^{\mathrm{a}}$ & 11 & 4 & 16 & 16 & 15 & 13 & 9 & Lp1 & 1 \\
\hline $03^{\mathrm{a}}$ & 1 & 4 & 3 & 5 & 11 & 1 & 15 & Lp14 & 4 \\
\hline $04^{\mathrm{a}}$ & 11 & 4 & 16 & 12 & 15 & 13 & 9 & Lp1(1)Lp14(1) & 2 \\
\hline
\end{tabular}

${ }^{a}$ New ST type assigned by SBT database of EWGIL.

3-parameter model by MEGA v5.05.

\section{Results}

\subsection{Legionella isolates}

A total of 55 isolates of Legionella were isolated from air conditioning cooling towers $(96.8 \%)$, fountains $(1.8 \%)$ and surface waters (1.8\%). Among them, $L$. pneumophila accounted for 29 isolates, approximately $52.7 \%$ of all isolates, whereas Legionalla species other than L. pneumophila accounted for $47.3 \%$ of the total. Among the 29 L. pneumophila species, the serotype 1 strains accounted for $44.8 \%$, whereas serotype 14 accounted for $55.2 \%$. L. feeleii was the dominant species (23.1\%) among 26 non-L. pneumophila species, followed by $L$. shakespearei and $L$. quateirensis, which severally accounted for $19.2 \%$. The above indicated that Legionella species were widely distributed in the public environment of Macau and L. pneumophila occupied the major proportion.

\subsection{Different prevalence of pathogenic genes in Legionella species}

In 29 L. pneumophila strains, flaA, pilE, asd, mip,
mompS, proA and neuA genes were $100 \%$ detected (Table 1). In 26 non-L. pneumophila, other genes included flaA, pilE, asd, mip, mompS, and proA which tested as $15.4 \%, 15.4 \%, 53.8 \%, 38.5 \%, 15.4 \%$ and $38.5 \%$. However, the neuA gene failed to amplify. The positive results of the three genes asd, mip and proA in non-L. pneumophila focused on the L. quateirensis and L. shakespearei strains.

For L. pneumophila strains, the results of real-time PCR were almost same as that of PCR, but the positive rate of flaA and mip genes were $93.1 \%$ and $96.5 \%$ (Table 1). The positive results for seven genes of non- $L$. pneumophila from real-time PCR exhibited some differences with that from PCR. Genes of flaA, asd, mip and proA showed a little lower sensitive while pilE, mompS and neuA genes were with more sensitive. In summary, the detection rates of seven genes were high in L. pneumophila strains and relatively low in non- $L$. pneumophila strains.

\subsection{Sequence-based typing}

The 29 environmental L. pneumophila strains could be divided into $10 \mathrm{STs}$ (Table 2), including 3 singletons, in which one was ST752 and the other two were new STs (ST01 and 02). According to the EWGLI SBT 
database submitted in March 2014, there were four new STs unique to Macao. The ST with the largest number of isolates was ST1 $(9 / 29,31.0 \%)$, followed by ST1417 and ST03 (4/29, 13.8\%), ST566 (3/29, $10.3 \%)$, then ST160, ST1119 and ST04 (2/29, 6.9\%). There were 36 alleles were identified across the 7 loci. At the individual loci level, mip gene provided the most alleles. Seven alleles were obtained from mip gene, whereas six alleles were obtained using flaA and neuA genes, five from the mompS gene, and four from pilE, asd and proA genes. The results revealed that ST1 was the main ST-type and mip gene showed greatest genetic diversity in Macau.

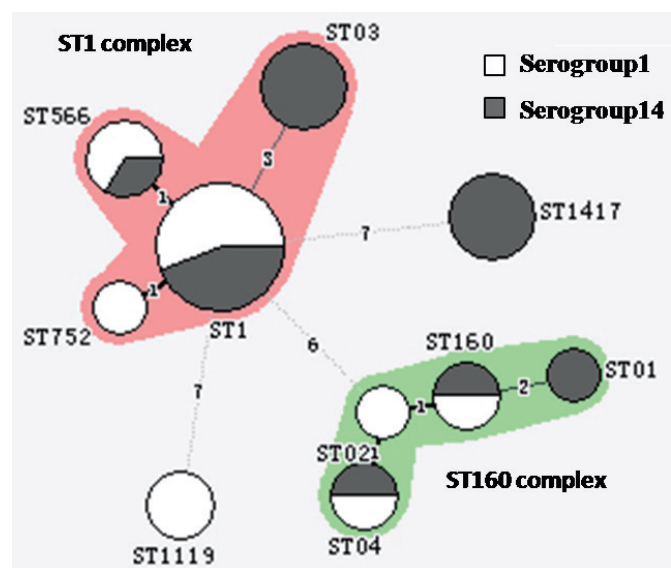

Figure 1. MST analysis of 29 L. pneumophila strains isolated in Macau. STs are represented by circles. The size of each circle indicates the number of isolates within this particular type; the STs are shown beside the circles and the number of isolates in each ST is shown in the circles. The branch distance is shown on the connecting line. This links STs or sets of STs with a branch distance less than or equal to 3 within a single ST complex.

\subsection{Population structure and Phylogenetic analysis}

All 10 STs identified from Macau were analysed for their population structure with the minimum spanning trees (Figure 1). Among them, 8 STs formed two clone complexes, and the other 2 different STs were determined to be singletons with four or more gene variants. The ST1 complex was a bigger clone group, including four STs groups containing 17 isolates. ST1 was suggested to be a primary founder, since there were 9 ST1 $(9 / 17,52.9 \%)$, the highest number of isolates in the complex. The other three STs in the ST1 complex were either single-locus variant or double-locus variants to ST1. In the other clone group ST160 complex, there were 4 STs with 6 isolates. Three (ST01, 02 and 04) of four new STs unique to Macao were found in this complex, while ST03 belonged to the ST1 complex.

The 29 strains in the study with the addition of 116 isolates from Guangzhou (10), and 52 isolated from Shenzhen (11) were put together and analysed again with minimum spanning tree by the BioNumerics software (Figure 2). They were identified as $47 \mathrm{STs}$, of which, 43 STs were grouped into eight clonal complexes. The other 4 STs (ST59, 114, 238, 1054) were singletons. Only ST1, ST 160 and ST752 were found in the three areas. The isolates from Macau were distributed mainly in two large clone complexes, I and II. Only one Macau STs could be found in the complexes III and VIII.

Clone complex I, the largest clone group in these three cities, contained the ST1 complex from Macau. There were four main STs, ST150, ST154, ST159 and ST160 in clone complex II, in which most of STs were single-locus or double-locus variants to ST154

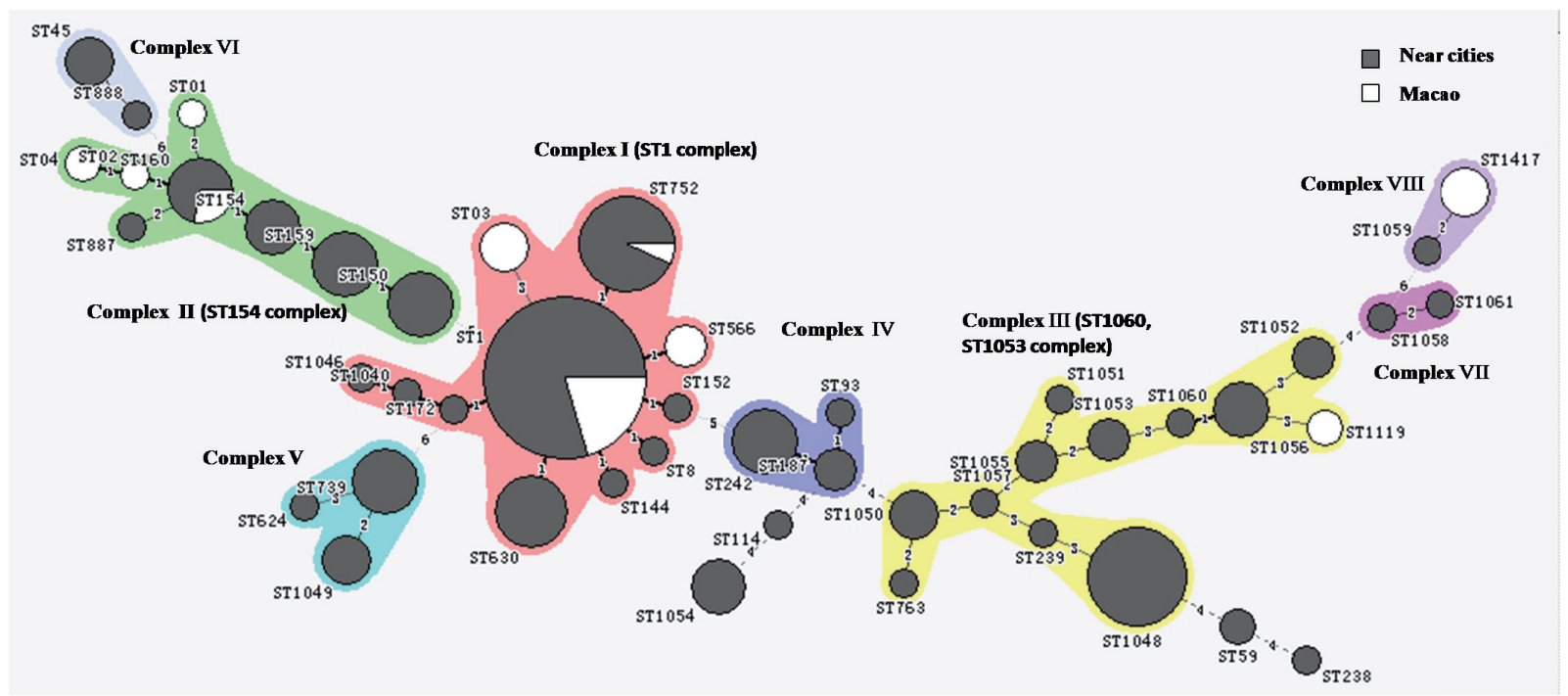

Figure 2. MST analysis of 197 L. pneumophila strains isolated from Macau, Guangzhou and Shenzhen. STs are represented by circles. The size of each circle indicates the number of isolates within this particular type; the STs are shown beside the circles. The heavy solid black lines connect two types within a single variant locus, the light solid black lines connect double-locus variants, the light solid gray lines connect triple-locus variants, the gray dashed lines connect quadruple-locus variants, and the dotted lines connect variants over quadruple-locus. The branch distance is shown on the connecting line. The colors of the halo surrounding the STs denote types that belong to the same clonal group. 


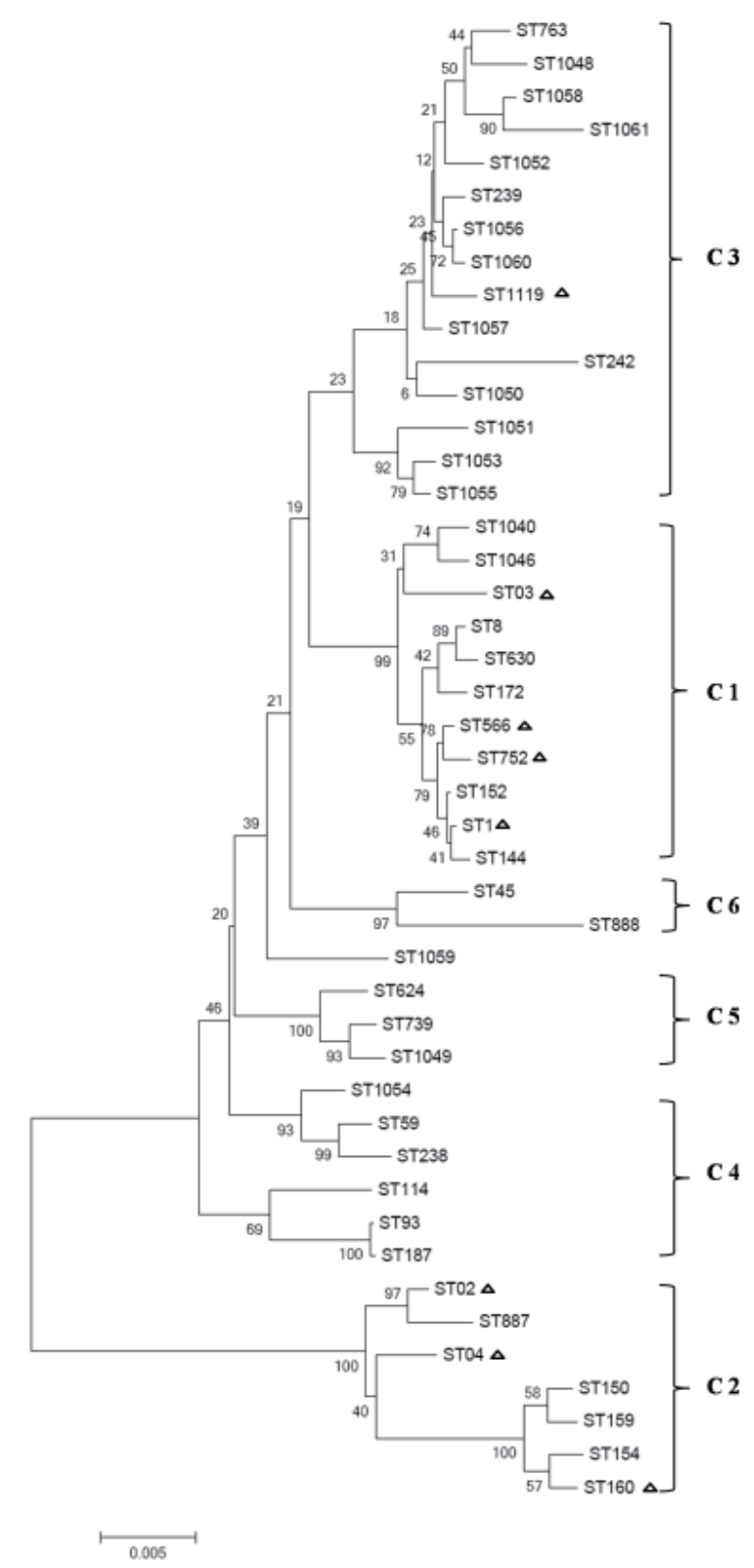

Figure 3. Phylogenetic analysis of the L. pneumophila STs isolated from Macau, Guangzhou and Shenzhen. The tree was constructed with MEGA v5.05. STs of strains isolated from Macao are labeled by black triangles. The scale bar indicates genetic distances between sequences. The percentages of replicate trees in which the associated STs clustered together in the bootstrap test are shown next to the branches. The evolutionary distances are in the units of the number of base substitutions per site.

or ST160. Clone complex III was another large group, which composed of the most STs with double-locus or triple-locus variants each other. The STs in the other four small clone complexes, IV to VII were from Shenzhen and Guangzhou, but not Macau.

STs phylogenetic analysis was conducted using a neighbor-joining method with the Tamura 3-parameter model based on concatenated sequences of SBT alleles. Because ST01 and ST1417 were obtained the neuA allele with new primers, the results of their phylogenetic analysis were unfaithful. The other
STs were divided into six groups (C1-C6) by the neighbor-joining analysis (Figure 3 ). These groups generally had a one-to-one correspondence with the six clonal complexes (complexes I to VI) determined by BioNumerics software. It was unexpected that the single C3 group identified by MEGA v5.05 contained both clonal complexes (III and VII). The distribution of Macau STs wasn't as wide as that of STs from near cities Guangzhou and Shenzhen, and ST1 was the preponderant STs among these three cities.

\section{Discussion}

We have previously studied the distribution and species of environmental Legionella isolates from Macau in detail (5). In this work, our main objective has been to analyze the prevalence of seven virulence genes of this species and reveal the STs distribution of $L$. pneumophila isolates from Macau. Furthermore, the L. pneumophila isolates from Macau were compared with the isolates from Guangzhou and Shenzhen. Our findings revealed the high prevalence of these seven virulence genes in $L$. pneumophila strains and low prevalence in non- $L$. pneumophila. Additionally, our study also indicated that STs had several unique allelic profiles and ST1 is the predominant ST-type in Macau.

The seven genes detected in this study are closely related to bacterial signal transduction, virulence, and adaptive capacity $(6,12-14)$. The different detection rates of these genes revealed the relationships between each virulence gene or combinations of these genes and the various species of Legionella in Macau. The high prevalence of these genes in L. pneumophila strains and low prevalence in non-L. pneumophila strains might reflect their own ability to adapt to the external environment and their strong pathogenicity to human beings. Some reports have shown that non- $L$. pneumophila could cause a variety of organ dysfunction (15). However, studies focused on detection of virulence genes associated with non-L. pneumophila strains are rarely found. This study represents the first detection for these seven virulence genes from both $L$. pneumophila and non-L. pneumophila strains which might provide a reliable basis to evaluate the pathogenic potential of the strains and their adaptability to the local environment.

In this study, the real-time PCR was also applied for its high level of efficiency and ability for specialization, as well as for its ability to multiply amplify the target genes and its ease of operation. The results from realtime PCR were generally consistent with that of PCR. The data suggested that real-time PCR, instead of conventional PCR, should be used to detect these seven genes, especially for large-scale investigation. If the PCR products needed to be collected for other purposes, or used to perform a test to compare and verify the relevant multi-alleles, both tests could be 
used together. Since the primers designed for real-time PCR were not appropriate for all L. pneumophila and non-L. pneumophila strains, a future study is needed to further investigate this issue.

These 29 L. pneumophila isolates were classified with the method recommended by EWGLI and four new STs were found unique to Macau. Within the 10 Macao STs, ST1 is the predominant ST-type (9/29, $31.0 \%$ ). The result was similar to the report in Japan where STI consisted of $29 \%$ of environmental isolates (16). In a recent study conducted in the United States, ST1 accounted for $25 \%$ and $49 \%$ of the number of the sporadic and environmental isolates, respectively (17). In one study conducted in England and Wales, ST1 was the most frequent STs, accounting for $35 \%$ of the number of L. pneumophila environmental isolates (18). From the L. pneumophila serogroup 1 isolates from potable systems, cooling towers, and hot springs in China, ST1 were reported to be about $14.3 \%$, to $53.1 \%$ and $92.3 \%$, respectively (19). For L. pneumophila strains, ST1 $(1,4,3,1,1,1)$ is predominant in environmental samples, widely distributed around world $(18,20,21)$. The Macau SBT analysis of L. pneumophila strains isolated from the public sites revealed the same ST-type characterization.

In the study, the loci mip and flaA offered more alleles in Macau's samples. The number of each allele presented by EWGLI from all over the world showed that the mompS locus provided the maximum number of alleles, neuA-Ah provided the next most, and mip, asd, proA and pilE, flaA provided the minimum. One report in South Korea showed that the mompS locus had the most alleles (2). Similarly, another study conducted in Canada obtained the same result that locus mompS provided the most alleles (22). However, one study on SBT of $L$. pneumophila strains in mainland China indicated that mip and flaA individually provided the most alleles in the isolates from cooling water and hot spring water (19). This might be attributed to the geographical correlation which could lead to the emergence of these results. Additionally, it might also reflect that the homology that exists between strains from Macau and mainland China.

Meanwhile, in order to understand the population structure and phylogeny of the L. pneumophlia strains isolated from Macau, the minimum spanning tree and the neighbor-joining method were respectively applied to compare Macau STs to those identified from nearby cities of Guangzhou and Shenzhen. All STs from these three cities were divided into eight clone complexes in the minimum spanning tree, and six groups could be seen by the neighbor-joining method. Consistent results from these two similar methods suggested that the phylogenetic analysis could be selected either individually or simultaneously. Among eight complexes generated in the minimum spanning tree, only four were related to Macau STs. The subtype distribution of L. pneumophlia strains isolated from Macau was not as extensive as that from other cities $(20,22,23)$. However, in complex I, ST1 from Macau was the major STs involved in, which reflects the concentration of local types. This supports that ST1 is the most common hereditary character of $L$. pneumophlia strains and extensively distributed all over the world. The majority of isolates from Macau kept also represented this distrubution. In this study, four new STs were found for the first time. It is convinced of that more new STs will be reported in further investigation. After a detailed study using phylogenetic analysis and population structure, the high diversity and specificity of L. pneumophlia strains isolated from Macau were observed. Since ST154 has been proven to be relevant to Legionnella epidemiology $(1,2)$, the closely connected ST04, ST02 and ST160, should bring significant attention and research interest.

To summarize, this study enables, for the first time, the ability to realize the prevalence of seven virulence genes of Legionella in Macau, to characterize the L. pneumophila environment isolates with SBT methodology, and to create a database of Macau's L. pneumophila profiles for use in epidemiological surveillance efforts. The findings of this study also contribute to the EWGLI-SBT database and to the knowledge of L. pneumophila diversity in southern China. Further studies are needed to reveal the relationships between each pathogenic gene or combinations of these genes and the pathogenicity of Legionella, and to analyze the correlation between environmental and clinical strains of Legionella.

\section{Acknowledgements}

This work was supported by a grant from the State Key Lab of Respiratory Disease, Guangzhou Medical College (2012-2014) and MACAO - Science and Technology Development Fund (039/2007/A3).We thank Miss Elizabeth Li for her great help with revising the article.

\section{References}

1. Helbig JH, Uldum SA, Bernander S, Lück PC, Wewalka G, Abraham B, Gaia V, Harrison TG. Clinical utility of urinary antigen detection for diagnosis of communityacquired, travel-associated, and nosocomial legionnaires' disease. J Clin Microbiol. 2003; 41:838-840.

2. Lee HK, Shim JI, Kim HE, Yu JY, Kang YH. Distribution of Legionella species from environmental water sources of public facilities and genetic diversity of L. pneumophila serogroup 1 in South Korea. Appl Environ Microbiol. 2010; 76:6547-6554.

3. Tsang KW, Ho PL, Ooi GC, et al. A cluster of cases of severe acute respiratory syndrome in Hong Kong. N Engl J Med. 2003; 348:1977-1985.

4. Guo J, Liang T, Hu C, Lv R, Yang X, Cui Y, Song Y, Yang R, Zhu Q, Song Y. Sequence types diversity of Legionella pneumophila isolates from environmental 
water sources in Guangzhou and Jiangmen, China. Infect Genet Evol. 2015; 29:35-41.

5. Zhao HB, Guan WD, Yang ZF, Lu X, Shi L, Cai F, Mo ZY. Detection and fatty acid analysis of public sites Legionella isolated from Macau. Ao Men Ke Ji Da Xue Xue Bao. 2011; 5:106-15. (in Chinese)

6. Cianciotto NP. Type II Secretion and Legionella Virulence. Curr Top Microbiol Immunol. 2013; 376:81102.

7. Fields BS, Benson RF, Besser RE. Legionella and Legionnaires' disease: 25 years of investigation. Clin Microbiol Rev. 2002; 15:506-526.

8. Gaia V, Fry NK, Afshar B, Lück PC, Meugnier H, Etienne J, Peduzzi R, Harrison TG. Consensus sequencebased scheme for epidemiological typing of clinical and environmental isolates of Legionella pneumophila. J Clin Microbiol. 2005; 43:2047-2052.

9. Ratzow S, Gaia V, Helbig JH, Fry NK, Lück PC. Addition of neuA, the gene encoding $\mathrm{N}$-acylneuraminate cytidylyl transferase, increases the discriminatory ability of the consensus sequence-based scheme for typing Legionella pneumophila serogroup 1 strains. J Clin Microbiol. 2007; 45:1965-1968.

10. Liang T. Population genetics analysis of Legionella pneumophlia isolated from Guangzhou by using sequence-based typing. M.Sc. Thesis. Liaoning University. 2012. (in Chinese)

11. Yuan M, Yuan YM, Yu MH, Duan Y. Sequenced-based typing research for Legionella pneumophila isolated from the cooling tower in Shenzhen. Huan Jing Yu Jiang Kang Za Zhi. 2010; 5:423-434. (in Chinese)

12. Khweek AA, Caution K, Akhter A, Abdulrahman BA, Tazi M, Hassan H, Majumdar N, Doran A, Guirado E, Schlesinger LS, Shuman H, Amer AO. A bacterial protein promotes the recognition of the Legionella pneumophila vacuole by autophagy. Eur J Immunol. 2013; 43:13331344.

13. Case CL, Kohler LJ, Lima JB, Strowig T, de Zoete MR, Flavell RA, Zamboni DS, Roy CR. Caspase-11 stimulates rapid flagellin-independent pyroptosis in response to Legionella pneumophila. Proc Natl Acad Sci U S A. 2013; 110:1851-1856.

14. Girard A, Roques E, Massie B, Archambault D. Flagellin in fusion with human rotavirus structural proteins exerts an adjuvant effect when delivered with replicating but non-disseminating adenovectors through the intrarectal route. Mol Biotechnol. 2014; 56:394-407.

15. Leggieri N, Gouriet F, Thuny F, Habib G, Raoult D, Casalta JP. Legionella longbeachae and endocarditis.
Emerg Infect Dis. 2012; 18:95-97.

16. Amemura-Maekawa J, Kikukawa K, Helbig JH, Kaneko S, Suzuki-Hashimoto A, Furuhata K, Chang B, Murai M, Ichinose M, Ohnishi M, Kura F. Distribution of monoclonal antibody subgroups and sequence-based types among Legionella pneumophila serogroup 1 isolates derived from cooling tower water, bathwater, and soil in Japan. Appl Environ Microbiol. 2012; 78:42634270.

17. Donohue MJ, O'Connell K, Vesper SJ, Mistry JH, King D, Kostich M, Pfaller S. Widespread molecular detection of Legionella pneumophila Serogroup 1 in cold water taps across the United States. Environ Sci Technol. 2014; 48:3145-3152.

18. Harrison TG, Afshar B, Doshi N, Fry NK, Lee JV. Distribution of Legionella pneumophila serogroups, monoclonal antibody subgroups and DNA sequence types in recent clinical and environmental isolates from England and Wales (2000-2008). Eur J Clin Microbiol Infect Dis. 2009; 28:781-791.

19. Qin T, Zhou H, Ren H, Guan H, Li M, Zhu B, Shao Z. Distribution of sequence-based types of Legionella pneumophila serogroup 1 strains isolated from cooling towers, hot spring, and potable water systems in China. Appl Environ Microbiol. 2014; 80:2150-2157.

20. Borchardt J, Helbig JH, Lück PC. Occurrence and distribution of sequence types among Legionella pneumophila strains isolated from patients in Germany: Common features and differences to other regions of the world. Eur J Clin Microbiol Infect Dis. 2008; 27:29-36.

21. Chasqueira MJ, Rodrigues L, Nascimento M, Marques T. Sequence-based and monoclonal antibody typing of Legionella pneumophila isolated from patients in Portugal during 1987-2008. Euro Surveill. 2009; 14:2935.

22. Reimer AR, Au S, Schindle S, Bernard KA. Legionella pneumophila monoclonal antibody subgroups and DNA sequence types isolated in Canada between 1981 and 2009: Laboratory Component of National Surveillance. Eur J Clin Microbiol Infect Dis. 2010; 29:191-205.

23. Underwood AP, Jones G, Mentasti M, Fry NK, Harrison TG. Comparison of the Legionella pneumophila population structure as determined by sequence-based typing and whole genome sequencing. BMC Microbiol. 2013; 13:302.

(Received June 1, 2015; Revised July 29, 2015; Rerevised August 9, 2015; Accepted August 9, 2015)

\section{Supplemental data}

Table S1. Sequence of primers designed for detecting virulence genes used in real-time PCR

\begin{tabular}{llc}
\hline Gene & Primers (5'-3') & Product size (bp) \\
\hline flaA & F: GATGCTACGTCTGCCTAT R: CCTGCGGTTCCACCTATT & 119 \\
pile & F: CGATGCTCATGCCACATT R: CCGTTCGGAGTTGTTGC & 120 \\
ssd & F: AAGCGGTTCATCTGGAGT R: TGCTGTGGGATAACTTGC & 119 \\
mip & F: AAATGCCATCGTTCCTG R: AGAAGCTGCGAAATCAGT & 164 \\
mompS & F: TGCCATCGTTCCTGAGTT R: GACCAGAAGCTGCGAAAT & 164 \\
proA & F: GGTGCTGTAGTTTCAACG R: GTGGCATTCTTACTGTGC & 143 \\
neu & F: TGCCTTGCAGTCGTCTTG R: TCCGTGGCTAAATCTTCC & 123 \\
\hline
\end{tabular}

Primer designed with the sequences downloaded from EWGLI (http://www.ewgli.org/) as a template. $F$ forward, $R$ reverse. 